\title{
Absorption of Electromagnetic Waves in Non-Dispersive Plasma due to Effect of Ponderomotive Force
}

\section{P. K. Thakur, S. Bhaila and J. J. Nakarmi}

Journal of Nepal Physical Society

Volume 6, Issue 1, June 2020

ISSN: 2392-473X (Print), 2738-9537 (Online)

Editors:

Dr. Binod Adhikari

Dr. Manoj Kumar Yadav

Mr. Kiran Pudasainee

JNPS, 6 (1), 123-130 (2020)

DOI: http://doi.org/10.3126/jnphyssoc.v6i1.30561

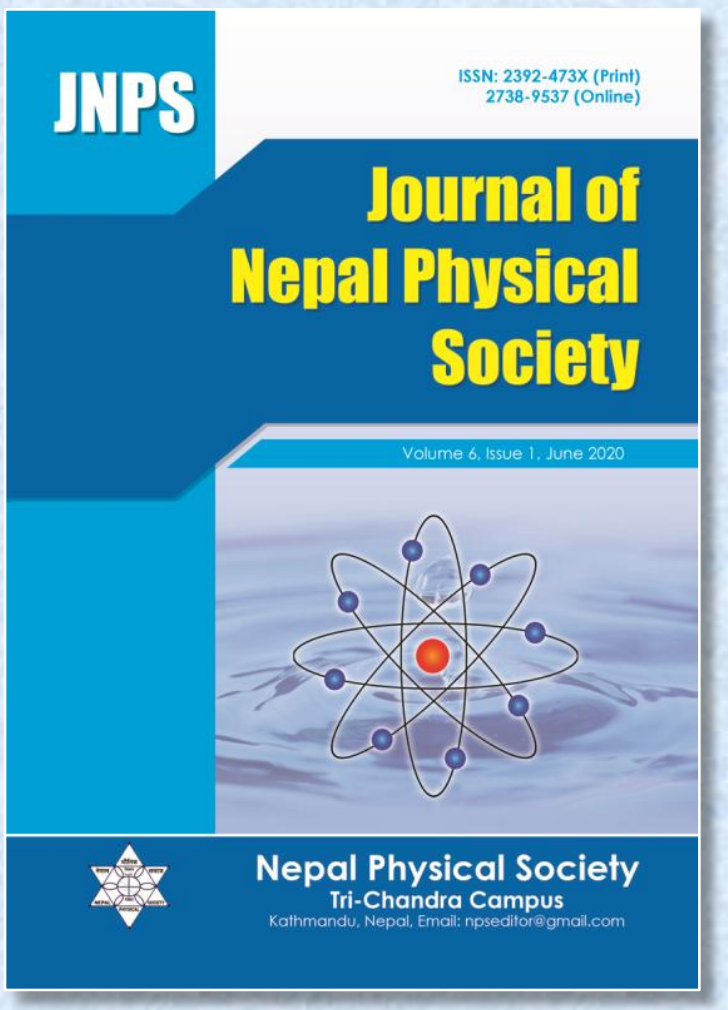

Published by:

Nepal Physical Society

P.O. Box: 2934

Tri-Chandra Campus

Kathmandu, Nepal

Email: npseditor@gmail.com 


\title{
Absorption of Electromagnetic Waves in Non-Dispersive Plasma due to Effect of Ponderomotive Force
}

\author{
P. K. Thakur, S. Bhaila and J. J. Nakarmi \\ Central Department of Physics, T. U., Kirtipur, Nepal \\ Corresponding Email: pk_thakur12@yahoo.com
}

Received: 28 Mar., 2020; Revised: 6 Jun., 2020; Accepted: 28 Jun., 2020

\begin{abstract}
The absorption coefficient of electromagnetic wave propagating through overdense absorbing plasma is calculated by using Fresnel's formula. Also, the force acting per unit volume of the electron fluid is calculated along $\mathrm{z}$ direction. The Fresnel's formula is reduced in terms of conductivity and angular frequency so that nature of absorption coefficient with respect to conductivity of plasma and angular frequency of wave is studied, with the assumption that conductivity and angular frequency are two independent terms. By keeping conductivity of plasma constant, it is seen that the absorption coefficient is increased with increase in angular frequency and also absorption coefficient has the lowest value for the highest value of conductivity. At constant angular frequency of incidence, the absorption coefficient is decreased with increase in conductivity and also absorption coefficient has the highest value of angular frequency of incidence. Also the nature of force on the plasma is studied by considering that the conductivity of plasma is very large with compare to angular frequency of incident laser. The expression of absorption coefficient is separated into real and imaginary parts, and then plots are studied with respect to these parts. It is found that in both cases, the force on plasma is existed for short in time and distance, and then damps exponentially. Also, the variation of potential with distance is cleared that the potential is shielded within multiple distance of Debye length.
\end{abstract}

Keywords: Absorption coefficient, angular frequency, conductivity, reflection coefficient, skin length.

\section{INTRODUCTION}

Light waves exert a radiation pressure which is very weak and hard to detect. For example, the comet tails formed by the pressure of sunlight is tainted by the added effect of particles streaming from the sun. When high powered microwaves or laser beams are used to heat or confine plasmas then the radiation pressure can reach several hundred thousand atmospheres. The force on plasma is coupled to the particles in a somewhat subtle way when it is applied, and is called the ponderomotive force [1]. Many nonlinear phenomena have a simple explanation in terms of the ponderomotive force [2].

To derive this nonlinear force, we consider the motion of an electron in the oscillating electromagnetic fields, $\boldsymbol{E}$ and $\boldsymbol{B}$, of a wave which is described by the equation of motion electron $[1,2]$ $m \frac{d \boldsymbol{v}}{d t}=-e[\boldsymbol{E}+\boldsymbol{v} \times \boldsymbol{B}]$

The nonlinearity comes partly from second order term because both $\boldsymbol{E}$ and $\boldsymbol{B}$ vanish in the equilibrium, so that the term is no larger than $v_{1} \times B_{1}$, where $v_{1}$ and $\boldsymbol{B}_{1}$ are the linear-theory values.

The wave equation in the electric field is,

$$
\boldsymbol{E}=\boldsymbol{E}_{s} \cos \omega t
$$

where $E_{s}$ contains the spatial dependence of electric field and $\omega$ is the angular frequency of the laser. Then the force becomes

$$
f_{N L}=-\frac{1}{4} \frac{e^{2}}{m \omega^{2}} \nabla \boldsymbol{E}_{s}^{2}
$$


This is the effective nonlinear force on a single electron. The force per unit volume is $f_{N L}$ times the electron density $\boldsymbol{n}_{e}$ and is given by,

$F_{N L}=n_{e} \times f_{N L}$

$F_{N L}=-\frac{\omega_{p}^{2}}{8 \pi \omega^{2}} \nabla\left\langle E^{2}\right\rangle$

where, $\omega_{p}^{2}=\frac{4 \pi n_{e} e^{2}}{m_{e}}$ is the plasma frequency.

The ponderomotive force is nonlinear force where a high frequency electromagnetic laser field induces by the plasma. This force is involved in many physical phenomena such as,

a. Momentum transfer to the target.

b. Self-focusing and filamentation of the laser beam

c. Plasma profile density changes such as the formation of caviton and soliton.

d. Parametric instability.

e. Second harmonic generation.

f. Magnetic field generation.

From the ratio of the ponderomotive force exerted on the ions $f_{p i}$ to the applied on the electron,

$\left(f_{p e}=f_{p}\right)$ is equal to $\frac{f_{p i}}{f_{p e}}=\frac{m_{e}}{m_{i}}$, where $m_{i}$ is

the mass of ion. However, the ponderomotive force exerted on the electrons is transmitted to the ions by the electric fields in the plasma because ponderomotive force on the ions is negligible. In this phenomenon the electrons are separated from the ions and an electric charge separation is created in the plasma [2].

\subsection{Effect of Ponderomotive Force on Wave Dispersion}

\subsubsection{Electron Wave Dispersion}

For the derivation of electron wave dispersion with ponderomotive force, we assume that

a. The problem is one- dimensional.

b. The ions are immobile.

c. The charge separation electric field is $\boldsymbol{E}$.

Here, the electrons are assumed to be an ideal gas and oscillations are adiabatic, then the electron pressure $\boldsymbol{P}_{\boldsymbol{i}}$ and electron density $\boldsymbol{n}_{\boldsymbol{e}}$ are related by equation of state $[2,3]$

$P_{i}=n_{e} k_{B} T_{e}$
From continuity and momentum equation of electrons

$$
\begin{aligned}
& \frac{\partial n_{e}}{\partial t}+\frac{\partial n_{e} v}{\partial x}=0 \\
& m_{e} n_{e}\left(\frac{\partial v}{\partial t}+v \frac{\partial v}{\partial x}\right)=-\frac{\partial P_{e}}{\partial x}-e n_{e} E+n_{e} f_{p}
\end{aligned}
$$

Consider the oscillating quantities are assumed to behave as sinusoidal, then the dispersion relation for the electron wave is

$$
\omega^{2}=\omega_{p}^{2}+\frac{3 k_{B} T_{e} k^{2}}{m_{e}}+i \frac{f_{p} k}{m_{e}}
$$

The last term on the right hand side of the Eqn (1.1.4) gives the ponderomotive force contribution to the wave dispersion. If we neglect ponderomotive force term then this equation changes to ordinary equation of electron plasma wave. Due to this term $\omega^{2}$, it is complex and wave growth fed by the imposed ponderomotive force is possible. At $T_{\theta}=0$ the real and imaginary parts are

$\omega_{R}=\omega_{p}^{2}+\frac{f_{p}^{2} k^{2}}{4 m_{e}^{2} \omega_{p}^{2}}$

and

$\omega_{I}=\frac{f_{p} k}{2 m_{e} \omega_{R}}$

It is interesting that even for $T_{e}=0_{\text {wave }}$ propagation occurs, and then the phase velocity $\nu_{\phi}$ and group velocity $v_{g}$ are given by

$$
\begin{aligned}
& v_{\phi}=\sqrt{\frac{\omega_{p}^{2}}{k^{2}}+\frac{i f_{p} k}{m_{e}}} \\
& v_{g}=\frac{i f_{p}}{2 m_{e}} / \sqrt{\omega_{p}^{2}+\frac{i f_{p} k}{m_{e}}}
\end{aligned}
$$

In the case without ponderomotive force, the group velocity vanishes if electron temperature becomes zero and wave doesn't propagate. So that dispersion relation for ion wave is at $T_{\theta}=0$ in the real and imaginary parts of $\omega$ are

$\omega_{R}=\sqrt{\frac{Z\left|f_{p} k\right|}{2 m_{i}}}$ and $\omega_{I}=\frac{Z \omega_{p} k}{2 m_{i} \omega_{R}}$ 
Here, ion wave can propagate even for the temperature becomes zero, unlike that in the case without ponderomotive force.

\section{THEORY}

When a laser is incident upon a solid at the same time a small fraction of it is absorbed and plasma is created at the surface and then laser energy can absorbed by the plasma so that it is heated to a very high temperature and the electron density gradient is increased towards the solid. Once the plasma is created, the laser energy can no longer be deposited upon to the solid target surface. The refractive index $\mu$ is given by the relation [4.5]

$\mu=\epsilon^{\frac{1}{2}}=\sqrt{1-\frac{\omega_{p}^{2}}{\omega^{2}}}$

Hence the laser light can only propagate up to a certain density, known as the critical density $\left(\mathrm{n}_{\mathrm{c}}\right)$ at which it is reflected. The surface of the plasma corresponding to this density is known as the critical surface. Critical density can be expressed as

$n_{c}=\frac{\omega_{p}^{2} m_{e}}{4 \pi e^{2}}$

In terms of the wavelength critical density is

$$
n_{c}=\frac{1.11 \times 10^{21}}{\lambda^{2}(\mu m)} \mathrm{cm}^{-2}
$$

As the absorption increases with respect to function of density, but the laser cannot propagate beyond the critical surface so we expect the temperature of the plasma to be maximum at the critical density. Between the critical surface and the solid, the temperature must therefore drop. Heat from the hot plasma at the critical surface is conducted down this temperature gradient, and this conducted heat generates more plasma at the solid surface, keeping the process going on. It can be seen that the temperature in the corona is roughly constant although the plasma is expanding in this region, this is somewhat compensated for the fact that some laser energy is being deposited here.

The absorption coefficient over a length $l$ is related to the difference between the incoming $l_{\text {in }}$ and outgoing $l_{\text {out }}$ laser intensity by

$$
\alpha_{i b}=\frac{I_{\text {in }}-I_{\text {out }}}{I_{\text {in }}}=1-\exp \left[\int_{0}^{l} k_{\text {ib }} d z\right]
$$

For a linear density profile of the form $n_{e}=n_{c}\left(1-\frac{z}{l}\right)$ and the analytical solution is given by

$\alpha_{i b}=1-\exp \left[-\frac{32}{15} \frac{v_{e i} n_{c} l}{c}\right]$.......

And for an exponential profile, we get

$\alpha_{i b}=1-\exp \left[-\frac{8}{3} \frac{v_{e i} n_{c} l}{c}\right]$

\subsection{Resonance Absorption}

We have to consider the interaction of p-polarized light of angular frequency $\omega$ on plasma with a density gradient. As the density varies as a function of $z$, so that the dielectric constant as a function of $z$ for the plasma $[6,12]$

$\in(z)=1-\frac{\omega_{p}^{2}}{\omega^{2}}$

It is cleared that when laser frequency is equal to the plasma frequency then $\varepsilon=0$ i.e. dielectric constant is zero, so that light will generate some part of resonance phenomena at the critical surface which can be seen by considering Poisson's equation, as plasma is neutral.

$\nabla \cdot(\in \boldsymbol{E})=0$

$\epsilon \nabla \cdot \boldsymbol{E}+\nabla \epsilon \cdot \boldsymbol{E}=0$

i.e. $\nabla . \boldsymbol{E}=-\frac{1}{\epsilon} \frac{\partial \epsilon}{\partial z} E_{z}(2.1 .1)$

As at critical surface $\varepsilon=0$ and according to this the gradient of the electric field becomes infinite at this point. In practice, this doesn't happen as we have treated $E$ as a real function but actually it has imaginary components due to Landau damping or collisional absorption and prevents the singularity. It is generally the case that the calculation of the electric field at the critical surface in the case of ppolarized light is non-trivial. We will thus first consider the interaction of s-polarized light with the plasma, where resonance absorption doesn't take place as the electric field oscillates along a direction where there is no gradient in density. Dielectric constant of the plasma and thus the way in which electromagnetic waves can propagate within it, is given by 


$$
\in=1+i \frac{4 \pi \sigma}{\omega}
$$

\subsection{Absorption coefficient of electromagnetic wave in non-dispersive plasma}

Let us assume that polarised laser beam is incident along the normal to the surface of the plasma i.e. $\mathrm{z}$ axis coincides with the direction of the normal [7]. The incident field components have the form

$$
E\left(E_{i n}, 0,0\right) ; B\left(0, B_{i n}, 0\right) \sim \operatorname{expi}\left(\mathrm{kz}-\omega_{0}\right)
$$

The intensity of radiation ionizes the atoms in the target in a period of the order of femtoseconds due to both field and electron ionization processes. The laser beam interacts with a homogeneous collision less plasma. The interaction process can be described by the set of Vlasov kinetic equation for the electrons and Maxwell's equation.

Thus, from Maxwell's equation,

$$
\nabla \times \boldsymbol{B}=\frac{4 \pi}{c} \boldsymbol{J}+\frac{1}{c} \frac{\partial \boldsymbol{E}}{\partial t}
$$

Taking curl of z-component

$$
\frac{\partial \boldsymbol{B}}{\partial z}=-\frac{4 \pi}{c} \boldsymbol{J}+i k_{0} \boldsymbol{E}
$$

And,

$$
\nabla \times \boldsymbol{E}=-\frac{1}{c} \frac{\partial \boldsymbol{B}}{\partial t}
$$

Taking y-component,

$$
\frac{\partial \boldsymbol{E}}{\partial z}=i k_{0} \boldsymbol{B}
$$

In vacuum the electric and magnetic fields are,

$\boldsymbol{B}=\boldsymbol{B}_{i n} \exp \left(-i \omega t+i k_{0} z\right)+\boldsymbol{B}_{R} \exp \left(-i \omega_{0} t+i k_{0} z\right)$

$\boldsymbol{E}=\boldsymbol{E}_{i n} \exp \left(-i \omega t+i k_{0} z\right)+\boldsymbol{E}_{R} \exp \left(-i \omega_{0} t+i k_{0} z\right)$

Where, $B_{\mathbb{i n}}$ and $B_{R}$ are the amplitudes of the incident and reflected waves respectively.

We know that reflection coefficient is given by the relation,

$$
R=\left|\frac{\boldsymbol{B}_{R}}{\boldsymbol{B}_{\text {in }}}\right|^{2}
$$

The magnetic field inside the plasma (medium) is

$$
\boldsymbol{B}_{p}=\boldsymbol{B}_{T} \exp \left(-i \omega_{0} t+i k_{0} z\right)
$$

Applying the boundary condition at the plasma vacuum interface,

$$
\begin{aligned}
& \boldsymbol{B}=\boldsymbol{B}_{p} \quad \text { at } \mathrm{z}=0 \\
& \frac{\partial \boldsymbol{B}}{\partial z}=\frac{\partial \boldsymbol{B}_{p}}{\partial z} \text { at } \mathrm{z}=0
\end{aligned}
$$

Applying boundary condition in Eqns (2.2.8) and (2.2.9)

We get,

$$
\begin{aligned}
& \boldsymbol{B}_{\text {in }}+\boldsymbol{B}_{R}=\boldsymbol{B}_{T} \\
& \boldsymbol{B}_{\text {in }}-\boldsymbol{B}_{R}=\frac{k}{k_{0}} \boldsymbol{B}_{T}
\end{aligned}
$$

After solving above equations

$$
\frac{\boldsymbol{B}_{R}}{\boldsymbol{B}_{\text {in }}}=\frac{k_{0}-k}{k_{0}+k}
$$

$\frac{\boldsymbol{B}_{R}}{\boldsymbol{B}_{\text {in }}}=\frac{1-\sqrt{\epsilon}}{1+\sqrt{\epsilon}}$

Applying this result we get the reflection coefficient as follows

$R=\left|\frac{1-\sqrt{\epsilon}}{1+\sqrt{\epsilon}}\right|^{2}$

And, absorption coefficient $\quad(A)=1-R$

$A=1-R=1-\left|\frac{1-\sqrt{\epsilon}}{1+\sqrt{\epsilon}}\right|^{2}$

$A=\frac{4 \sqrt{\epsilon}}{|1+\sqrt{\epsilon}|}$

\subsection{Ponderomotive force in non- dispersive plasma}

Here, we have taken a local relation between field and current at a certain medium [11]

$\boldsymbol{J}=\sigma \boldsymbol{E}$

$\in=1+i \frac{4 \pi \sigma}{\omega_{0}}$

Where, $\sigma$ is the conductivity and $\epsilon$ is the dielectric permeability. 
Here, we have obtained the space dependence of the fields inside the plasma

$$
\boldsymbol{E}=\epsilon^{-\frac{1}{2}} \boldsymbol{B}
$$

And,

$$
\boldsymbol{B}=B_{p} \exp (i q z)
$$

Where,

$$
\boldsymbol{B}_{p}=\frac{B_{i n}}{1+\epsilon^{-\frac{1}{2}}}
$$

Using the relation for ponderomotive force [7]

$$
\begin{aligned}
& \boldsymbol{F}_{p}=\frac{1}{c} \boldsymbol{J} \times \boldsymbol{B}=\frac{\sigma}{c \epsilon^{-1 / 2}} \exp 2\left(i q z-i \omega_{0} t\right) \\
& =\frac{\sigma}{c \in^{-1 / 2}} \frac{4 B_{i n}^{2}}{\left(1+\epsilon^{-1 / 2}\right)^{2}} \exp \left(2 i q z-2 i \omega_{0} t\right)
\end{aligned}
$$

For high conductivity plasma,

$$
\in \approx i \frac{4 \pi \sigma}{\omega_{0}}
$$

It is convenient to introduce,

$\epsilon^{\frac{1}{2}}=\frac{1+i}{\delta}$

Where,

$$
\delta=\sqrt{\frac{\omega_{0}}{2 \pi \sigma}}<<1
$$

Then, we have

$1+\epsilon^{-\frac{1}{2}}=1+\frac{\delta}{1+i}=1+\frac{\delta}{1+i} \frac{1-i}{1-i}=\frac{2+\delta}{2}-i \frac{\delta}{2}$

$\left(1+\epsilon^{\frac{1}{2}}\right)^{2}=1+\delta$ neglecting $\delta^{2}$

And,

$\in \epsilon^{*}=\frac{1+i}{\delta} \frac{1-i}{\delta}=\frac{2}{\delta^{2}}$

Thus,

$$
|\epsilon|=\sqrt{\epsilon}=\frac{\sqrt{2}}{\delta}
$$

From all above the equations, we can obtain the force acting per unit volume of the electron fluid by considering only in the inward $z$ direction for the plasma

$$
F_{z}=4 B_{i n}^{2} \frac{\sigma}{c} \frac{\delta}{1+\delta} \exp \left(2 i k_{0} \in^{1 / 2} z-2 i \omega_{0} t\right)
$$

Again, solving for $\delta$

$\delta=\frac{1+i}{\epsilon^{1 / 2}}$

$|\delta|^{2}=\frac{1+i}{\epsilon^{1 / 2}} \frac{1-i}{\epsilon^{1 / 2}}=\frac{2}{\epsilon}=2 \frac{\omega_{0}}{i 4 \pi \sigma}=-i \frac{\omega_{0}}{2 \pi \sigma}$

Taking magnitude only

$\delta=\sqrt{\frac{\omega_{0}}{2 \pi \sigma}}$

Solving, $\left|\epsilon^{\frac{1}{2}}-1\right|^{2}$ and $\left|\epsilon^{\frac{1}{2}}+1\right|^{2}$ separately,

$1-\epsilon^{\frac{1}{2}}=1-\frac{1+i}{\delta}=\frac{\delta-1}{\delta}-\frac{i}{\delta}$

$\left|\epsilon^{\frac{1}{2}}-1\right|^{2}=\left(\frac{\delta-1}{\delta}-\frac{i}{\delta}\right) \times\left(\frac{\delta-1}{\delta}+\frac{i}{\delta}\right)=\frac{2(1-\delta)}{\delta^{2}}$

$$
\text { (2.3.10) }
$$

Similarly,

$\left|\epsilon^{\frac{1}{2}}+1\right|^{2}=\frac{2(1+\delta)}{\delta^{2}}$

Substituting these values, the reflection coefficient becomes

$$
\begin{aligned}
& R=\frac{\frac{2(1+\delta)}{\delta^{2}}}{\frac{2(1+\delta)}{\delta^{2}}}=-1+\frac{2}{(1+\delta)} \\
& 1+R=\frac{2}{(1+\delta)} \\
& (1+\delta)=\frac{2}{1+R}
\end{aligned}
$$

Introducing the skin length for the normal skin effect as[10], 
$l_{s}=\frac{\delta}{k_{0}}=\left(\frac{\omega_{\mathrm{o}}}{2 \pi \sigma}\right)^{\frac{1}{2}} \times \frac{c}{\omega_{0}}$

After solving,

$2 i k_{0} \in^{\frac{1}{2}} z=2 k_{0} \frac{i-1}{\delta} z$

Substituting all these values, Eqn (2.3.8) becomes

$$
F_{z}=B_{i n}^{2} \frac{1+R}{l_{s} \pi} \exp \left[2(i-1) \frac{z}{l_{s}}-2 i \omega_{0} t\right]
$$

Here, force is expressed in separated form for real and imaginary terms

$$
\begin{aligned}
& F_{\text {real }}=B_{\text {in }}^{2} \frac{1+R}{l_{s} \pi} \exp \left(-\frac{2 z}{l_{s}}\right) \\
& F_{\text {im }}=B_{\text {in }}^{2} \frac{1+R}{l_{s} \pi} \exp \left[-2\left(\omega_{0} t-\frac{z}{l_{s}}\right)\right]
\end{aligned}
$$

In both the cases the exponential terms contain negative terms.

And absorption coefficient

$$
\begin{aligned}
& A=1-R=\frac{2 \delta}{1+\delta} \\
& A=2 \frac{\omega_{0}^{1 / 2}}{(2 \pi \sigma)^{\frac{1}{2}}+\omega_{0}^{1 / 2}}
\end{aligned}
$$

This is required expression for absorption coefficient of laser plasma in terms of conductivity $\sigma$ and angular frequency $\omega_{0}$, which is derived by using Fresnel's formula. Here we have considered that $\omega_{0}$ and $\sigma$ are two independent terms.

\section{RESULTS AND DISCUSSION}

3.1 Variation of Absorption coefficient of the laser plasma with respect to conductivity and frequency:

The absorption coefficient for an electromagnetic wave with frequency $\omega_{0}$ and conductivity $\sigma$ of plasma is given by the relation, (2.3.17)

$$
A=2 \frac{\omega_{0}^{1 / 2}}{(2 \pi \sigma)^{\frac{1}{2}}+\omega_{0}^{1 / 2}}
$$

Here we have derived formula for $\omega_{0} \ll \sigma$.

\subsubsection{When Conductivity is constant}

From the variation of absorption coefficient with incident laser frequency for a given value of conductivity, it is observed that, for increase in some value of frequency of laser, the absorption coefficient increases and then saturates. For our purpose we have chosen the value of conductivity stated in [8] for $\mathrm{z}=1$. The different curves are for the conductivity $\sigma_{1}=5.9 \times 10^{18} / \mathrm{s}, \sigma_{2}=6 \times 10^{19} / \mathrm{s}$ and $\sigma_{3}=6 \times 10^{20} / \mathrm{s}$. It is seen that when frequency increases the absorption coefficient also increases.

$$
i, e_{x} A \propto \sqrt{\omega_{0}}
$$

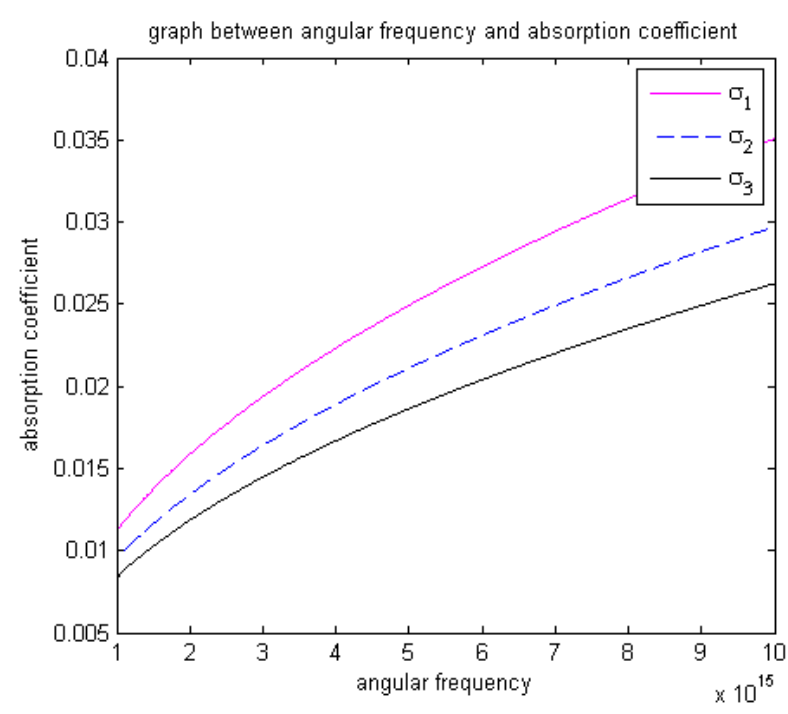

Fig. (3.1): Graph between frequency of laser and absorption coefficient

From above graph it is cleared that the variation of absorption coefficient is highest for the conductivity with lowest value. This is due to the fact that we have considered $\sigma \gg \omega_{0}$ but here angular frequency is approaching to the conductivity.

\subsubsection{When Frequency is Constant}

Here, we have considered plasma with variable conductivity. Three separate waves with frequency $\omega_{01}=10^{11} / s, \quad \omega_{02}=10^{12} / s$, and $\omega_{03}=10^{12} / s$ are plotted with variable values of conductivity. For plasma with variable conductivity, the absorption coefficient decreases with increase in conductivity also it is cleared that the wave with highest 
frequency have the highest absorption coefficient. Also the magnitude of absorption coefficient is very low for the frequency whose magnitude is very low with that of conductivity.

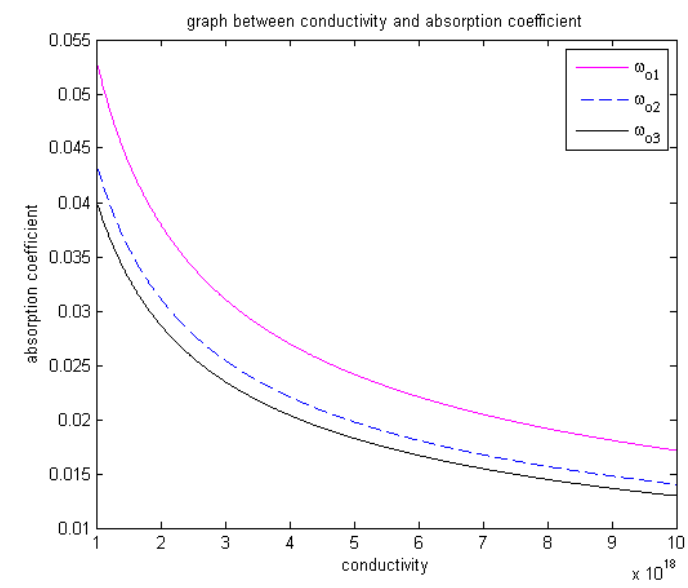

Fig. (3.2): Graph between conductivity and absorption coefficient

3.2 Variation of ponderomotive force of real and imaginary parts with respect to distance and time:

3.2.1 Variation of real part of the force with respect to distance:

From the above relation of pondermotive force in the plasma along $\mathrm{z}$-axis is

$$
F_{z}=B_{i n}^{2} \frac{1+R}{l_{s} \pi} \exp \left[2(i-1) \frac{z}{l_{s}}-2 i \omega_{0} t\right]
$$

After substituting the value of $1+\mathrm{R}$ and $\mathbb{I}_{a}$ in the above equation, and for $\delta \ll 1$, then the real part of the ponderomotive force becomes

$$
F_{z \text { (real })}=\frac{B_{i n}^{2}}{\pi} 2 \frac{\left(2 \pi \sigma \omega_{0}\right)^{\frac{1}{2}}}{c} \exp \left(-\frac{2 z\left(2 \pi \sigma \omega_{0}\right)^{\frac{1}{2}}}{c}\right)
$$

The pondermotive force for an electromagnetic wave with distance along $\mathrm{z}$-axis is plotted in fig (3.3). We have plotted the curve with incident amplitude in 0.5 unit and then it is plotted for three different values of laser frequency. Here we have considered for only one value of conductivity. From the graph it is cleared that the force decreases exponentially and exist for few hundred of micron. Here the force is decreased more exponentially for laser with the highest value of frequency, and as if the frequency is decreased then the value of decreasing rate is also decreased.

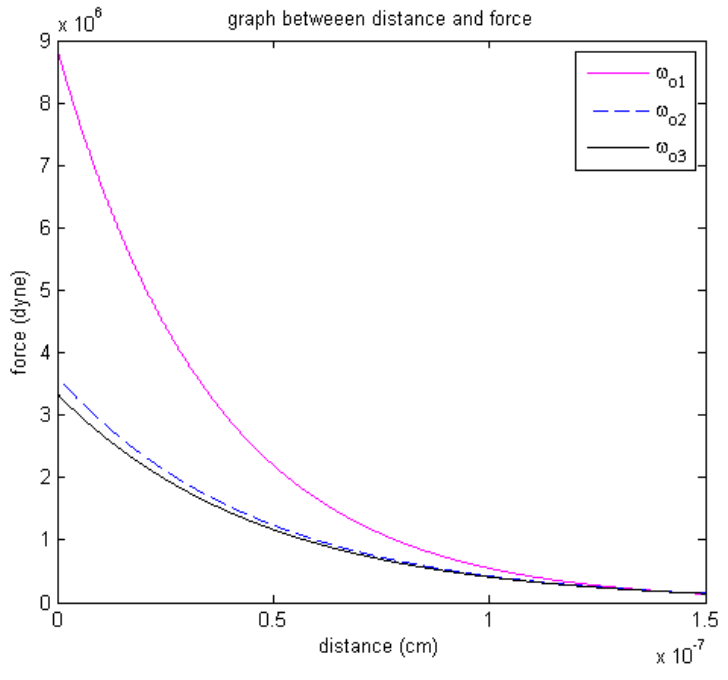

Fig. (3.3): Graph between real part of pondermotive force and distance

\subsubsection{Variation of imaginary part of the force with respect to time:}

The imaginary part of the ponderomotive force in the plasma due to laser passing through it, is given by the relation

$$
F_{z(i m g)}=\frac{B_{i n}^{2}}{\pi} 2 \frac{\left(2 \pi \sigma \omega_{0}\right)^{\frac{1}{2}}}{c} \exp \left(-2\left(\omega_{0}-\frac{z}{l_{s}}\right)\right)
$$

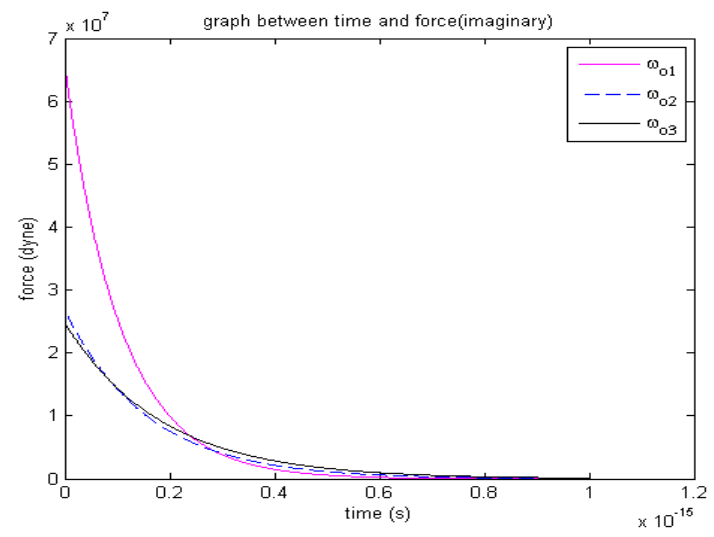

Fig. (3.4): Graph between imaginary part of ponderomotive force and time

This is the imaginary part of the force in the distance $\mathbb{R}_{g}$ from the plane at $\mathrm{z}=0$, where $\mathbb{l}_{g}=\frac{\mathbb{E}}{\left(2 \pi \sigma \mathbb{w}_{0}\right)^{\frac{1}{2}}}$. The graph of fig (3.4) is cleared that the variation of force in the three different values of laser frequency with respect to time, in addition, it exists only for a very small value, i.e. a small 
fraction of nanosecond. Further it is seen that, the force exponential decrease in the highest level for the laser with the highest value of frequency.

\subsection{Variation of potential of the laser plasma with respect to Distance:}

From the relation between potential of plasma and distance is $[2,9]$

$$
\frac{\Phi(x)}{\Phi}=\exp \left(-\frac{x}{\lambda_{D}}\right)
$$

The graph between the ratio of potential in the laser plasma with respect to distance is as shown in fig (3.5) in which the ratio of potential is decreased exponentially with distance. From the graph we can also say that the potential is shielded within few multiple distance of Debye length and for a typical Cs plasma the Debye length is $1.05 \times 10^{-1} \mathrm{~cm}[1]$.

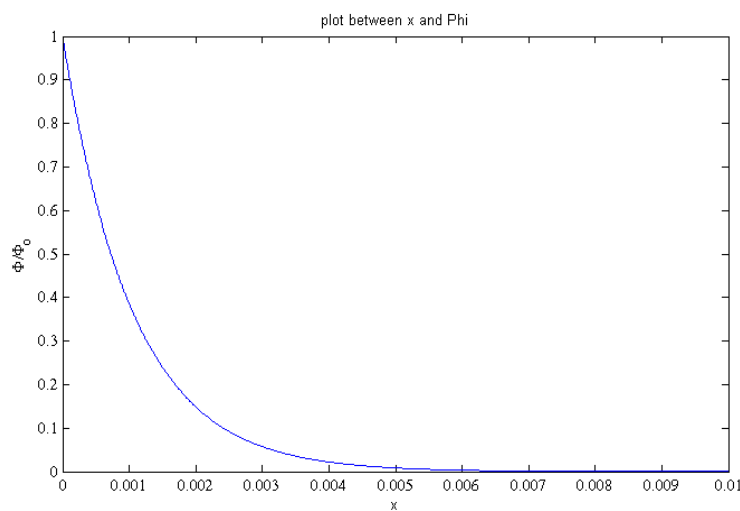

Fig (3.5): Graph between potential with distance

\section{CONCLUSIONS}

In this work we have considered the very basic and classical absorption mechanism when the intensity of radiation is low, also its theoretical calculation and graphical representation are studied. The theoretical value of absorption coefficient is showed the relation with angular frequency of radiation and conductivity of plasma. Then the result is cleared that the absorption coefficient increases with increase in angular frequency and also absorption coefficient increases with decrease in conductivity. The expression of ponderomotive force in terms of real and imaginary parts is signified that the force decreases exponentially with distance and time. Finally, the variation of potential of plasma with distance is studied and from the graph it is cleared that the potential is decreased exponentially with distance. On deriving the expression of absorption coefficient and ponderomotive force, we have assumed that the conductivity and angular frequency of radiation are two independent terms because conductivity is very large in compare to angular frequency but in fact they have relation so interested can study the relation for them at the range near to conductivity and angular frequency or absorption coefficient with their mutual relation.

\section{AKNOWLEDGEMENTS}

The author wishes to acknowledge the useful discussions with Prof. Dr. Lok Narayan Jha and Prof. Dr. Raju Khanal for providing some important materials, and Head of Central Department of Physics, Prof. Dr. Binil Aryal for providing an excellent research environment and motivation.

\section{REFERENCES}

[1] Chen, F. F., Introduction to Plasma Physics and Controlled Fusion, Vol-I, Second edition, Plenum Press, New York (1984).

[2] Eliezer, S. The Interaction of High-power Laser with Plasmas, Plasma Physics and Controlled Fusion, 45(2), 181-181, (2003).

[3] Flazner, S. P. An Introduction to Inertial Confinement Fusion, 1st Edition, Boca Raton, CRC Press, Florida, USA, (2006).

[4] Ginberg, V, L. The Propagation of Electromagnetic wave in Plasmas, 2nd Edition edition, Pergamon Press, United Kingdom (1970).

[5] Kruer, W. L. Physics of Laser Plasma Interaction, McGraw-Hill, Pennsylvania, USA (1988).

[6] Wark, J. Plasma physics of Short Pulse, Oxford Press, University of Oxford (2010).

[7] Parker, S. E. and Lee W. W. A fully nonlinear characteristic method for gyrokinetic simulation, Physics of Fluids B: Plasma Physics 5, 77 (1993).

[8] Nakarmi, J. J. Study of Magnetic Field Generation and its Effects on the Energy transport in the Corona of Laser fusion Plasma, Ph D Thesis, Tribhuvan University, Nepal (1999).

[9] Krapchev, V. B., Kinetic Theory of the Ponderomotive Effects in a Plasma, Phys. Rev. Lett. 42, 497 (1979).

[10] Rozmus, W., Tikhonchuk, V. T., A model of ultrashort laser pulse absorption in solidtargets, Phys. Plasma 3, 360 (1996).

[11] Jackson, J. D., Classical Electrodynamics, $3^{\text {rd }}$ edition, Wiley India (2011).

[12] Pearlman, J. S., Thompson, J. J., Max, C.E., Polarization-Dependent Absorption of Laser Radiation Incident on Dense-Plasma Planar Targets Phys. Rev. Lett.,38,1397(1977). 